\title{
Ontogeny of adrenal steroid biosynthesis: why girls will be girls
}

\author{
Perrin C. White \\ Department of Pediatrics, University of Texas Southwestern Medical Center, Dallas, Texas, USA.
}

\begin{abstract}
Male and female external genitalia appear identical early in gestation. Testosterone exposure at 8-12 weeks' gestation causes male differentiation. Female fetuses virilize if their adrenals secrete excessive levels of androgens, as occurs in congenital adrenal hyperplasia due to 21-hydroxylase deficiency. This can be ameliorated by administering dexamethasone to the mother. A study by Goto et al. in this issue of the JCI provides a rationale for this treatment by demonstrating that the fetal hypothalamic-pituitary-adrenal axis is fully functional when the genitalia differentiate (see the related article beginning on page 953). Dexamethasone suppresses this axis, reducing abnormal secretion of adrenal androgens. Their results also show that cortisol synthesis by the fetal adrenal decreases after this period, allowing the adrenal to secrete high levels of dehydroepiandrosterone, an androgen precursor. However, this does not virilize female fetuses because androgens are aromatized to estrogens in the placenta. Thus normal sexual differentiation requires exquisite timing of fetal cortisol and androgen secretion versus placental capacity for aromatization.
\end{abstract}

Early in gestation, the external genitalia are anatomically identical in both sexes. Female external genitalia represent the default state with male differentiation occurring if high levels of testosterone (as secreted by fetal testes) induce steroid $5 \alpha$-reductase type 2 (SRD5A2) and are then converted to dihydrotestosterone in genital skin. The critical period for such differentiation is $8-12$ weeks post conception (wpc). Later exposure to testosterone increases growth of the penis or clitoris, but does not induce fusion of labia majora and minora into scrotum and penile urethra, respectively. Differentiation of the genitalia can also be influenced by adrenal steroid biosynthesis. The early ontogeny of such biosynthesis has now been studied by Goto et al. (1) in this issue of the JCI (a preliminary report of some of these findings was published previously; see ref. 2).

\section{Endocrinology of human pregnancy}

To place this work in context, consider the endocrinology of human pregnancy (Figures

Nonstandard abbreviations used: CYP21, cytochrome P450 21-hydroxylase; DHEA, dehydroepiandrosterone; DHEAS, dehydroepiandrosterone sulfate; HPA, hypothalamic-pituitary-adrenal; HSD3B, $3 \beta$-hydroxysteroid dehydrogenase; wpc, weeks post conception.

Conflict of interest: The author has declared that no conflict of interest exists.

Citation for this article: J. Clin. Invest. 116:872-874 (2006). doi:10.1172/JCI28296.
1 and 2). During early gestation, the estradiol required to maintain pregnancy is provided by the corpus luteum in the maternal ovary. But after 8 weeks' gestation, most estradiol is synthesized by the fetoplacental unit (3). Dehydroepiandrosterone sulfate (DHEAS) is secreted by the fetal adrenal cortex. Synthesis of DHEAS requires importation of precursor cholesterol into mitochondria, regulated by the steroidogenic acute regulatory (StAR) protein, followed by conversions mediated by the cholesterol side-chain cleavage enzyme (CYP11A), 17 $\beta$-hydroxylase/17,20lyase (CYP17) and steroid sulfotransferase (SULT2A1). In the placenta, steroid sulfatase (also known as arylsulfatase, ARSC1) converts DHEAS back to dehydroepiandrosterone (DHEA), which is converted to androstenedione by $3 \beta$-hydroxysteroid dehydrogenase type 1 (HSD3B1; an isozyme distinct from the HSD3B2 expressed in the adrenal cortex). Androstenedione is aromatized to estrone by CYP19 and then converted to estradiol by HSD17B1.

Additionally, DHEAS is oxidized in the fetal liver to $16 \alpha-\mathrm{OH}$ DHEAS, which is converted by the placenta to estriol by the same enzymes that are involved in estradiol synthesis. Although estriol has little known functional significance, it does not circulate in the blood of nonpregnant women at high levels and is therefore a marker of function of the fetoplacental unit.
Thus DHEAS is the main steroid secreted by the fetal adrenal cortex during mid-gestation (Figure 2). Fetal cortisol is required later in pregnancy for lung maturation, particularly surfactant production, but this cannot be allowed to occur too early because amniotic fluid surfactant levels may control the timing of parturition (4). Maternal cortisol cannot normally reach the fetus because it is oxidized to cortisone, an inactive steroid, by HSD11B2 in the placenta (5). A low ratio of fetal cortisol to DHEAS secretion is maintained by decreased expression of adrenal HSD3B2, which is required for cortisol biosynthesis.

Placental aromatase prevents female fetuses (as well as their mothers) from virilization by the large amounts of DHEAS secreted by the fetal adrenal cortex. Conditions under which placental aromatase activity is decreased (including congenital deficiencies of aromatase or its accessory enzyme, P450 oxidoreductase; ref. 6), are associated with fetal and maternal virilization. Placental aromatase can also protect female fetuses from virilization when the mothers themselves have high circulating testosterone levels resulting from poorly controlled congenital adrenal hyperplasia due to CYP21 deficiency (7). This observation implies that high placental aromatase expression too early in pregnancy might interfere with normal male differentiation, but then how is the female fetus protected from virilization before placental aromatase expression has reached high levels?

To answer this question, Goto et al. (1) examined expression of the enzymes required for cortisol biosynthesis in fetuses at 7-10 wpc; previously, the youngest human fetuses studied were 14 weeks old (8). The authors found that adrenal explants from 8-wpc fetuses have a robust capacity to secrete cortisol, and that this secretion is responsive to adrenocorticotrophic hormone (ACTH). By $10 \mathrm{wpc}$, cortisol secretion begins to decrease. Thus they propose that cortisol secretion is relatively 


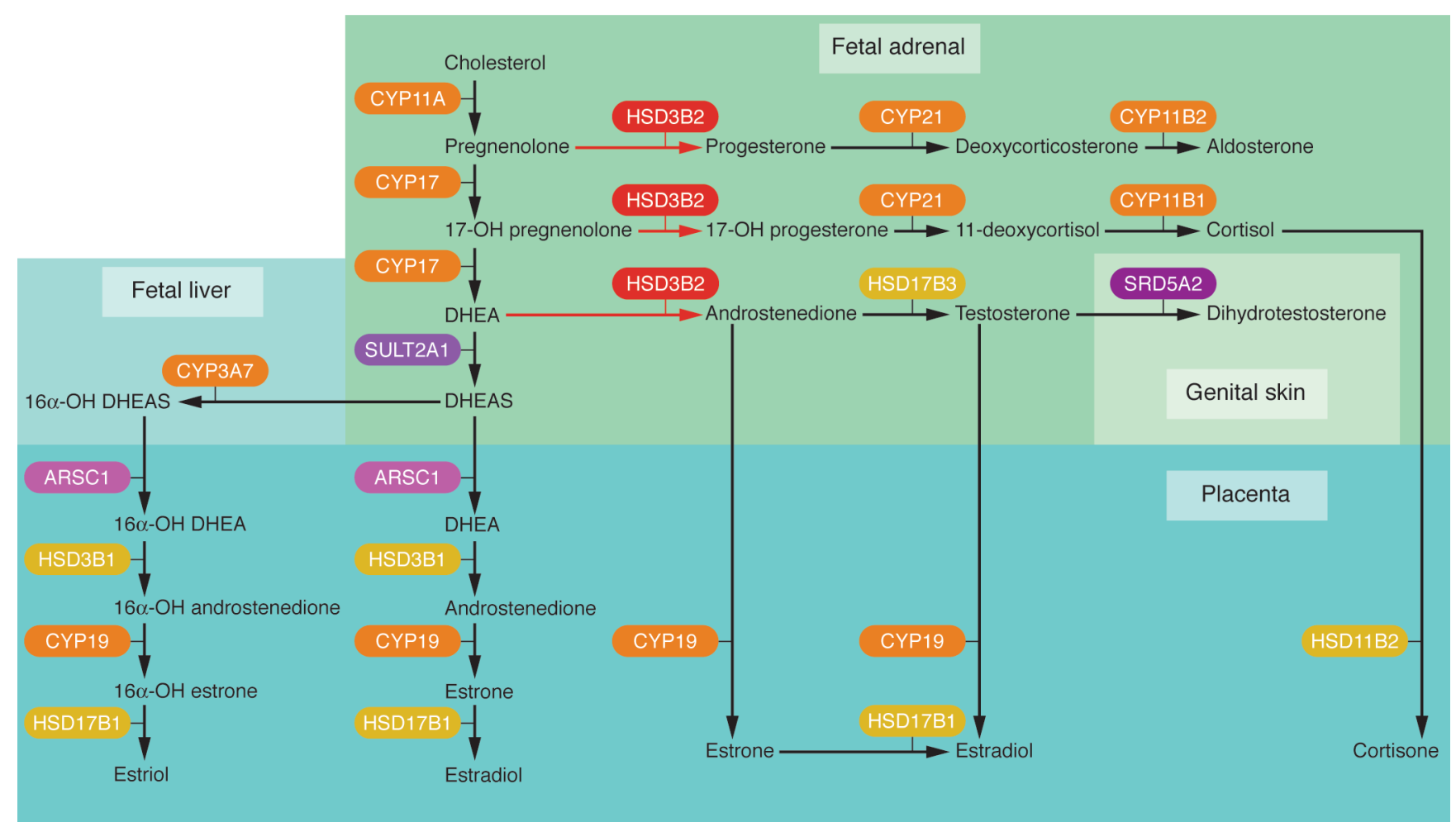

\section{Figure 1}

Steroid biosynthesis and metabolism during gestation. Conversions within the fetal adrenal cortex, fetal liver, male (i.e., testosterone-exposed) genital skin, and placenta are denoted by arrows; the enzyme mediating each conversion is also shown. Relative amounts of cortisol and DHEAS secreted by the fetal adrenal cortex are regulated by expression of HSD3B2, which is labeled in red. ARSC1, arylsulfatase; SRD5A2, steroid $5 \alpha$-reductase type 2; SULT2A1, steroid sulfotransferase.

high during the period when DHEAS secretion would interfere with genital development in female fetuses (Figure 2). The high level of cortisol production suppresses the fetal hypothalamic-pituitary-adrenal (HPA) axis, keeping DHEAS production at relatively low levels.

Later, HSD3B2 expression decreases, decreasing cortisol secretion, reducing HPA axis suppression, and increasing DHEAS secretion. Near the end of gestation, HSD3B2 expression increases and remains high until late in childhood. It decreases again at adrenarche, increasing DHEA secretion and leading to development of pubic and axillary hair (9).

\section{Prenatal treatment of congenital adrenal hyperplasia}

By demonstrating that there is indeed a functioning fetal HPA axis when the external genitalia are differentiating, the present work (1) provides a rationale for prenatal treatment of congenital adrenal hyperplasia due to CYP21 deficiency. Patients with this condition cannot synthesize cortisol efficiently (10). This decreases negative feedback on the hypothalamus and pituitary, thus increasing production of steroids proximal to the blocked conversion of 17-hydroxyprogesterone to 11-deoxycortisol. These precursors are shunted into adrenal androgen biosynthesis, increas- ing secretion of androstenedione; this is converted into testosterone by HSD17B5 in the adrenal gland (the presence of this enzyme in the critical period is demonstrated by Goto et al. in ref. 1) and by this and other HSD17B isozymes in other tissues. Testosterone then causes genital ambiguity in affected females. Although maternal cortisol cannot cross the placenta due to placental HSD11B2, the potent synthetic glucocorticoid dexamethasone is not readily metabolized by this enzyme (its highly electrophilic $9 \alpha$-fluorine atom draws negative charge from its $11 \beta$-hydroxyl group, rendering a hydride transfer - a necessary step of the 11-dehydrogenase reaction -

\section{Figure 2}

Relative levels of cortisol and DHEAS secretion by the fetal adrenal cortex during gestation as well as postnatally. Approximate times of several events are shown (see "Endocrinology of human pregnancy"). The existence of the first peak of cortisol secretion at 8-10 wpc was demonstrated in this issue by Goto et al. (1). Vertical axis is logarithmic, but values are approximate. Horizontal axis is not to scale.

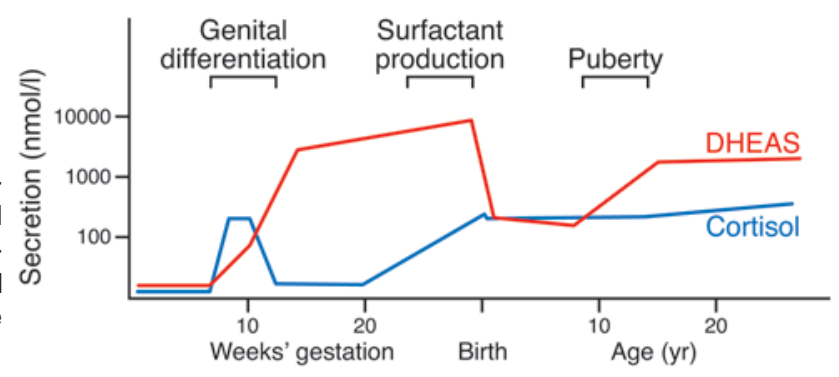


energetically unfavorable). Thus dexamethasone suppresses the fetal HPA axis when administered to the mother. This intervention reduces virilization of the genitalia in affected females, but must be started before the genitalia begin to virilize at 8 weeks' gestation $(11,12)$. Because prenatal diagnosis by chorionic villus sampling is not possible until several weeks later, all at-risk pregnancies must be treated, with therapy subsequently discontinued in all male and unaffected female pregnancies. Maternal side effects of dexamethasone administration include signs of Cushing syndrome such as excessive weight gain and cutaneous striae. Adverse effects on the fetus are apparently limited, but animal studies suggest potential consequences of prenatal glucocorticoid exposure including increased risk of metabolic syndrome (13, 14). Although delineation of risks versus benefits requires further study, it seems desirable to use the minimum effective dexamethasone dosage.

\section{Implications}

The study reported by Goto et al. (1) implies that high doses of dexamethasone are most necessary in the relatively narrow time window when cortisol levels would normally be high and the genitalia are differentiating. Elevated androgens later in pregnancy should have less effect due to placental aromatase and the completed differentiation of the genitalia. Indeed, a reduced dexamethasone dose of $5 \mu \mathrm{g} / \mathrm{kg} / \mathrm{d}$ after 23 weeks' gestation effectively prevented prenatal virilization in a single case (15).
Therefore reducing dexamethasone doses after perhaps 16 weeks' gestation (to allow for uncertainty in dating conception) might decrease adverse maternal effects without compromising efficacy. Testing this hypothesis would be best accomplished with a prospective, randomized controlled trial, which might well need to be multinational to have adequate power (16).

The work of Goto et al. (1) illustrates how normal sexual differentiation requires exquisite coordination of the development of the HPA axis, secretion of cortisol and androgen precursors by the fetal adrenal cortex, placental capacity for aromatization, and developmental events within the external genitalia.

\section{Acknowledgments}

The author's work is supported by the Audrey Newman Rapoport Distinguished Chair in Pediatric Endocrinology. The author thanks Walter Miller for helpful discussion.

Address correspondence to: Perrin C. White, Department of Pediatrics, UT Southwestern Medical Center, 5323 Harry Hines Boulevard, Dallas, Texas 753909063, USA. Phone: (214) 648-3501; Fax: (214)648-9772; E-mail: Perrin.white@ utsouthwestern.edu.

1. Goto, M., et al. 2006. In humans, early cortisol biosynthesis provides a mechanism to safeguard female sexual development. J. Clin. Invest. 116:953-960. doi:10.1172/JCI25091.

2. Goto, M., et al. 2002. Steroidogenic enzyme expression within the adrenal cortex during early human gestation. Endocr. Res. 28:641-645.
3. Simpson, E.R., and MacDonald, P.C. 1981. Endocrine physiology of the placenta. Annu. Rev. Physiol. 43:163-188.

4. Mendelson, C.R., and Condon, J.C. 2005. New insights into the molecular endocrinology of parturition. J. Steroid Biochem. Mol. Biol. 93:113-119.

5. White, P.C., Mune, T., and Agarwal, A.K. 1997. 11ßhydroxysteroid dehydrogenase and the syndrome of apparent mineralocorticoid excess. Endocr. Rev. 18:135-156.

6. Fluck, C.E., et al. 2004. Mutant P450 oxidoreductase causes disordered steroidogenesis with and without Antley-Bixler syndrome. Nat. Genet. 36:228-230.

7. Lo, J.C., et al. 1999. Normal female infants born of mothers with classic congenital adrenal hyperplasia due to 21-hydroxylase deficiency. J. Clin. Endocrinol. Metab. 84:930-936.

8. Narasaka, T., Suzuki, T., Moriya, T., and Sasano, H. 2001. Temporal and spatial distribution of corticosteroidogenic enzymes immunoreactivity in developing human adrenal. Mol. Cell. Endocrinol. 174:111-120.

9. Gell, J.S., et al. 1998. Adrenarche results from development of a 3beta-hydroxysteroid dehydrogenasedeficient adrenal reticularis. J. Clin. Endocrinol. Metab. 83:3695-3701.

10. Speiser, P.W., and White, P.C. 2003. Congenital adrenal hyperplasia. N. Engl. J. Med. 349:776-788.

11. New, M.I., et al. 2001. Prenatal diagnosis for congenital adrenal hyperplasia in 532 pregnancies. J. Clin. Endocrinol. Metab. 86:5651-5657.

12. Forest, M.G., Morel, Y., and David, M. 1998. Prenatal treatment of congenital adrenal hyperplasia. Trends Endocrinol. Metab. 9:284-289.

13. [Anonymous]. 2002. Consensus statement on 21hydroxylase deficiency from the Lawson Wilkins Pediatric Endocrine Society and the European Society for Paediatric Endocrinology. J. Clin. Endocrinol. Metab. 87:4048-4053.

14. Seckl, J.R., and Miller, W.L. 1997. How safe is longterm prenatal glucocorticoid treatment? JAMA. 277:1077-1079.

15. Coleman, M.A., and Honour, J.W. 2004. Reduced maternal dexamethasone dosage for the prenatal treatment of congenital adrenal hyperplasia. BJOG. 111:176-178.

16. Lajic, S., Nordenstrom, A., Ritzen, E.M., and Wedell, A. 2004. Prenatal treatment of congenital adrenal hyperplasia. Eur. J. Endocrinol. 151:U63-U69. 


\title{
Cardiac 7-transmembrane-spanning domain receptor portfolios: diversify, diversify, diversify
}

\author{
Stephen B. Liggett
}

Cardiopulmonary Genomics Program, University of Maryland School of Medicine, Baltimore, Maryland, USA.

\begin{abstract}
Enhanced signaling in myocytes by the $G$ protein $G_{q}$ has been implicated in cardiac hypertrophy and the transition to heart failure. $\alpha_{1}$-Adrenergic receptors $\left(\alpha_{1}\right.$-ARs) are members of the 7-transmembrane-spanning domain (7-TM) receptor family and signal via interaction with $G_{q}$ in the heart. The specific effects of a loss of $\alpha_{1}$-AR signaling in the heart are explored by O'Connell et al. in this issue of the JCI (see the related article beginning on page 1005). Paradoxically, gene ablation of the $\alpha_{1 \mathrm{~A}}$ and $\alpha_{1 \mathrm{~B}}$ subtypes in mice results in a maladaptive form of reactive cardiac hypertrophy from pressure overload, with a predisposition to heart failure. Thus signaling to the $\alpha_{1}$-AR (compared with signaling from other receptors such as angiotensin receptors, which also couple to $G_{q}$ ) appears to be specifically required for a normal hypertrophic response. This represents another example of how receptors that share common $G$ proteins have diversified, developing unique signaling programs. These findings may have particular clinical relevance because of the widespread use of $\alpha_{1}$-AR antagonists in the treatment of hypertension and symptomatic prostate enlargement.
\end{abstract}

It is now recognized that there are 2 distinct subgroups of $\alpha$-adrenergic receptors, designated as $\alpha_{1}$-adrenergic receptors ( $\alpha_{1}$-ARs) and $\alpha_{2}$-ARs, all of which are members of the superfamily of 7-transmembrane-spanning domain (7-TM) receptors (also termed $\mathrm{G}$ protein-coupled receptors). There are 3 human $\alpha_{1}$-AR subtypes, denoted $\alpha_{1 \mathrm{~A}}, \alpha_{1 \mathrm{~B}}$, and $\alpha_{1 D}$. Since $\alpha_{1}$-ARs expressed on vascular smooth muscle act to constrict and thus increase peripheral vascular resistance, there has been substantial development and widespread use of $\alpha_{1}$-AR antagonists for the treatment of hypertension. What has not been well acknowledged is the fact that $\alpha_{1}$-ARs are also expressed on cardiomyocytes, and thus treatment of hypertension with $\alpha_{1}$-AR antagonists may also have effects on the heart that are distinct from afterload reduction. All $\alpha_{1}$-AR subtypes couple to the heterotrimeric $G$ protein $\mathrm{G}_{\mathrm{q}}$. Upon agonist activation, the $\mathrm{G}_{\alpha}$ subunit activates the effector phospholipase C, which produces at least 2 intracellular second messengers, inositol-1,4,5-tri-

Nonstandard abbreviations used: AR, adrenergic receptor; $\mathrm{AT}_{1}$, angiotensin II type 1; TAC, transverse aortic constriction; 7-TM, 7-transmembrane-spanning domain.

Conflict of interest: The author has declared that no conflict of interest exists.

Citation for this article: J. Clin. Invest. 116:875-877 (2006). doi:10.1172/JCI28234. phosphate and diacylglycerol. The former increases intracellular calcium, while the latter activates several PKC isoenzymes that modify heart failure (1). Since catecholamines are elevated in heart failure, cardiac $\alpha_{1}-A R / G_{q}$ signaling is activated to various extents in the syndrome.

The $G_{\alpha q}$ pathway has been studied extensively as to its role in cardiac hypertrophy and heart failure (2). Cardiac overexpression of $G_{\alpha q}$ in transgenic mice (3) results in hypertrophy, decreased ventricular function, loss of $\beta$-adrenergic receptor inotropic responsiveness, and induction of a classic hypertrophy gene expression profile. In these mice, pressure overload by surgical transverse aortic constriction (TAC), pregnancy, or higher transgenic overexpression of $\mathrm{G}_{\alpha \mathrm{q}}$ resulted in cardiomyocyte apoptosis and decompensated heart failure (3, 4). Other studies showed that transgenic overexpression of a $\mathrm{G}_{\alpha \mathrm{q}}$ dominant-negative minigene resulted in the lack of a hypertrophy response to TAC (5). Furthermore, cardiac overexpression of a constitutively activated $\alpha_{1 \mathrm{~B}}$-AR resulted in cardiac hypertrophy (6), while a more severe cardiomyopathy developed as a result of overexpression of the $\mathrm{G}_{\mathrm{q}}$-coupled angiotensin II type $1\left(\mathrm{AT}_{1}\right)$ receptor $(7)$. These studies, then, began to point toward hyperactive $G_{\alpha q}$ signaling as a key mechanism causing hypertrophy, depressed ventricular function, and failure.
A readily drawn conclusion from such studies might be that in the human heart, factors that increase $G_{\alpha q}$ signaling predispose to cardiac hypertrophy and, potentially, the transition from hypertrophy to decompensated heart failure. In addition, approaches that decrease this signaling might be protective against the development of heart failure or be beneficial in treatment.

\section{Ablation of $\alpha_{1}$-ARs and cardiac hypertrophy}

In the report by O'Connell et al. in this issue of the JCI (8), the hypertrophic response to TAC was assessed in mice in which the genes encoding $\alpha_{1 \mathrm{~A}}$-AR and $\alpha_{1 \mathrm{~B}}$-AR had been ablated ( $\alpha_{1 \mathrm{~A} / \mathrm{B}} \mathrm{KO}$ mice). Mice without these $G_{\alpha q}$-coupled receptors demonstrated rapid decompensation and heart failure after TAC. In those that survived, echocardiographic studies showed lower ejection fractions than in WT mice. Although both sets of mice exhibited hypertrophy, the $\alpha_{1 \mathrm{~A} / \mathrm{B}} \mathrm{KO}$ mice had increased apoptosis and interstitial fibrosis. Furthermore, they had an atypical hypertrophy-associated gene profile, with minimal changes in expression of $\beta$-myosin heavy chain, $\alpha$-skeletal actin, and atrial natriuretic factor transcripts. These data suggest that $\alpha_{1}-A R / G_{q}$ signaling is necessary for adaptation to pressure overload. This issue is of substantial clinical importance because of the extensive use of $\alpha_{1}$-AR antagonists for the treatment of hypertension and symptomatic prostate enlargement. In a large cohort of hypertensive patients, those treated with the $\alpha_{1}$-AR antagonist doxazosin had a relative risk of $2.04(95 \%$ confidence interval $=1.79-2.32$, $P<0.001)$ of developing heart failure compared with those receiving a diuretic (9). Other studies with smaller cohorts have also observed this relationship but indicate an attenuation of this risk after adjustment for systolic blood pressure (10). Of note, this latter study found that systolic blood pressure was lower in the diuretic group compared with the $\alpha_{1}$-AR antagonist group, particularly in women, in whom the risk of heart failure was greatest. This may 